\title{
Seroprevalence of Five Zoonotic Pathogens in Wild Ruminants in Xinjiang, Northwest China
}

\author{
Jian-Yong Wu, Jian-Jun Li, ${ }^{1}$ Deng-Feng Wang,, Yu-Rong Wei,, Xiao-Xiao Meng,, \\ Gunuer Tuerxun, ${ }^{1}$ Hongduzi Bolati, ${ }^{1}$ Kang-Kang Liu, ${ }^{2}$ Masha Muhan, ${ }^{3}$ \\ Ayiqiaolifan Shahan, ${ }^{3}$ Dilireba Dilixiati, ${ }^{3}$ and Xue-Yun Yang ${ }^{1}$
}

\begin{abstract}
Wild ruminants are at risk for zoonotic pathogen infection as a result of interactions with domestic animals and humans. One way to assess the level of a wild ruminant disease in a population is to determine the seroprevalence of the pathogen of interest. The objective of this study was to determine the seroprevalence of five zoonotic pathogens in wild ruminants in Xinjiang, Northwest China. In 2009 and 2011-2015, 258 wild ruminant sera samples were collected from various species. Samples were obtained from 30 Siberian ibexes, 94 goitered gazelles, 6 Tibetan antelopes, 32 argali sheep, 16 roe deer, 20 blue sheep, 56 red deer, and 4 wild yaks, in 10 regions of Xinjiang. Samples were tested using antibodies against Brucella spp., Chlamydophila abortus, Coxiella burnetii, Toxoplasma gondii, and West Nile virus. Seropositivity was detected for all five pathogens, with detection rates of Brucella spp., C. abortus, C. burnetii, T. gondii, and West Nile virus of $2.3 \%(95 \%$ confidence interval [CI], 0.5-4.2\%), 6.2\% (95\% CI, 3.3-9.1\%), 7.8\% (95\% CI, 4.5-11.0\%), 2.3\% (95\% CI, 0.5$4.2 \%$ ), and $0.8 \%$ (95\% CI, 0-1.8\%), respectively. The level of pathogens differed for different species and different regions. The results indicate that seropositivity to zoonotic pathogens is common among wild ruminants in Xinjiang, Northwest China, with $C$. burnetii and $C$. abortus detected at the highest levels. This study provides a baseline for future assessment of spillover events.
\end{abstract}

Keywords: wild ruminants, $C$. burnetii, $C$. abortus, zoonotic disease, seroprevalences

\section{Introduction}

$\mathbf{Z}$ OONOTIC DISEASES CAN pass from wild or domestic animals to humans (Miernyk et al. 2019). Most emerging pathogens causing human disease are zoonotic pathogens (Belay et al. 2017). More than $60 \%$ of emerging infectious disease events are caused by zoonotic pathogens (Petrosillo 2019), which were originally discovered in wildlife and pose serious public health risks worldwide (Escribano-Romero et al. 2015, Menachery et al. 2016, Li et al. 2017, Tomassone et al. 2018).

Wild ruminants include a variety of animal species, have a wide distribution in mountain and desert areas, and serve as an important wild reservoir of a variety of pathogens. Previous studies showed that wild ruminants can carry pathogens, such as avian influenza virus, Brucella spp., and West
Nile virus, and can provide a bridge for the transmission of pathogens from wild animals into domestic livestock and even humans (Jennelle et al. 2016, Muñoz et al. 2019, Steyn et al. 2019). When a pathogen spills into wildlife, it can form a reservoir in wildlife, significantly limiting the ability for humans to control its spread.

As an important habitat for wild ruminants, Xinjiang is located on the northwestern border of China, adjacent to Central Asia, Russia, Pakistan, and Mongolia. Xinjiang encompasses several geographical forms, such as mountains, deserts, basin, and grasslands, and is a potential hotspot for infectious disease outbreaks. Peste des Petits Ruminants virus and avian influenza virus have been detected in wild ruminants in this region (Wei et al. 2016, Xia et al. 2016), but there have been limited reports about other pathogens, especially those able to transmit from wildlife to domestic livestock or

\footnotetext{
${ }^{1}$ Institute of Veterinary Medicine, Xinjiang Academy of Animal Sciences, Urumqi, China.

${ }^{2}$ School of Public Health, Sun Yat-sen University, Guangzhou, China.

${ }^{3}$ Wildlife Focus Disease Monitoring Station of Xinjiang, Urumqi, China.
} 
Table 1. Characteristics of Sera Collected from Wild Ruminants

\begin{tabular}{|c|c|c|c|c|c|c|c|c|}
\hline Animal & Argali sheep & Blue sheep & Goitered gazelle & Red deer & Roe deer & Siberian ibex & Tibetan antelopes & Wild yak \\
\hline 2009 & 7 & 2 & 21 & 22 & 7 & 14 & - & - \\
\hline 2011 & 2 & - & 1 & - & - & 1 & - & - \\
\hline 2012 & - & 1 & 4 & - & 1 & - & 6 & 4 \\
\hline 2013 & 13 & 10 & 15 & 16 & 2 & 15 & - & - \\
\hline 2014 & 6 & 7 & 30 & 12 & 6 & - & - & - \\
\hline 2015 & 4 & - & 23 & 6 & - & - & - & - \\
\hline
\end{tabular}

- , serum was not collected.

humans. In this study, we collected sera from eight species of wild ruminants and determined the seroprevalence of five zoonotic diseases: Brucella spp., West Nile virus, Chlamydophila abortus, Coxiella burnetii, and Toxoplasma gondii. The results of this study will provide reference data to study the transmission of pathogens from or into wildlife populations.

\section{Materials and Methods}

The study was approved by the Xinjiang Forestry Department. Sera samples were collected and processed according to the Law of The People's Republic of China for the Protection of Wildlife (http://www.npc.gov.cn/wxzl/gongbao/2016-08/ 22/content_1995643.htm). Sample collection was carried out in 2009, and 2011-2015 (Table 1). Samples from a total of 258 wild ruminants were collected from 10 regions (Aksu, Altay, Bayingolin, Changji, Hami, Ili, Khotan, Kashgar, Tacheng, and Urumqi) in Xinjiang, Northwest China. The samples included 32 argali sheep (Ovis ammon), 20 blue sheep (Pseudois nayaur), 94 goitered gazelles (Gazella subgutturosa), 56 red deer (Cervus elaphus), 16 roe deer ( $\mathrm{Ca}$ preolus pygargus), 30 Siberian ibex (Capra sibirica), 6 Tibetan antelopes (Pantholops hodgsonii), and 4 wild yaks
(Bos mutus) (Fig. 1 and Supplementary Appendix Table S1). Animal species identification was performed by experts. Blood samples of $\sim 3 \mathrm{~mL}$ were collected by jugular vein blood sampling from each ruminant, and allowed to clot at room temperature for 1 or $2 \mathrm{~h}$. Clots were removed by centrifuging at $2000 \times g$ for $5 \mathrm{~min}$ in a refrigerated centrifuge. All the samples were then transported and shipped within $72 \mathrm{~h}$ to the laboratory in sterile collection tubes with refrigeration at $-20^{\circ} \mathrm{C}$, and were then stored at $-80^{\circ} \mathrm{C}$ before testing.

All sera samples were tested for the presence of antibodies specific to Brucella spp., C. abortus, C. burnetii, T. gondii, and West Nile virus using the ID Screen Brucellosis Serum Indirect Multi-species Kit (IDVet, Grabels, France), the C. abortus Antibody Test Kit (IDEXX, Hoofddorp, Netherland), the Q-fever (C. burnetii) Antibody Test Kit (IDEXX), the ID Screen Toxoplasmosis Indirect MultiSpecies Kit (IDVet), and the ID Screen West Nile Competition kit (IDVet, Montpellier, France), respectively, according to the manufacturer's instructions.

Descriptive statistics were used to determine the seroprevalence of the five zoonotic pathogens at the individual animal level, and 95\% confidence intervals (CIs) were calculated. Data were analyzed using SPSS software version

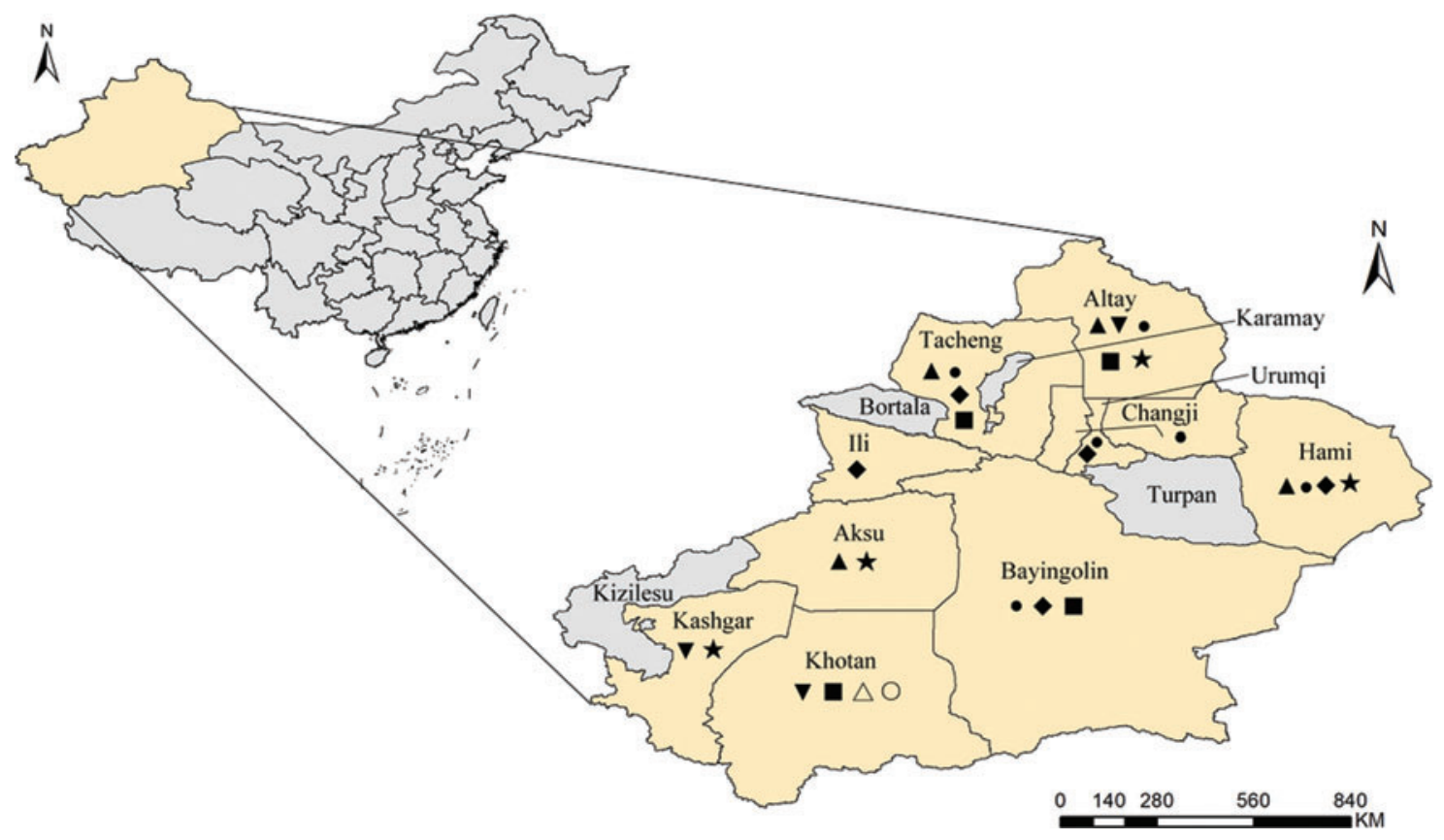

FIG. 1. Geographic distribution of sera collection. The yellow indicates where sera samples were collected. $\boldsymbol{\Delta}$, argali sheep; $\boldsymbol{\nabla}$, blue sheep; $\bullet$, goitered gazelle; $\bullet$, red deer; $\boldsymbol{\square}$, roe deer; $\star$, Siberian ibex; $\triangle$, Tibetan antelope; $\bigcirc$, wild yak. Color images are available online. 
23.0 (IBM, Chicago). The chi-squared test was used to analyze correlations between different zoonotic diseases, and $p$ values $<0.05$ were considered statistically significant.

\section{Results}

Antibodies were detected in all five of the investigated pathogens in wild ruminants. Of these, 56 wild ruminants carried one pathogen, accounting for $21.7 \%$ (56/258), and no animals carried two or more pathogens. Of the samples with positive antibody signals, the seropositivity ranged from $0.8 \%(95 \% \mathrm{CI}, 0-1.8 \%)$ for West Nile virus, $2.3 \%(95 \%$ CI, $0.5-4.2 \%$ ) for both Brucella spp. and T. gondii, $6.2 \%$ (95\% CI, 3.3-9.1\%) for C. abortus, and 7.8\% (95\% CI, $4.5-$ $11.0 \%$ ) for $C$. burnetii. There was greater seropositivity for C. abortus and C. burnetii infection than for Brucella spp., $T$. gondii, and West Nile virus $(p<0.05)$. Eight wild ruminant species were tested with no seropositivity for the five pathogens detected in the roe deer and wild yak samples (Fig. 2).

To prevent disease spread, it is important to understand the animal distribution of zoonotic pathogens. Six positive samples showed seropositivity to Brucella spp.; these samples were obtained from two goitered gazelles, two red deer, and two Tibetan antelopes, with respective detection rates of $2.6 \%$ (95\% CI, 0-5.0\%), 3.6\% (95\% CI, 0-8.4\%), and 33.3\% (95\% CI, 4-78\%). None of the Siberian ibexes, argali sheep, roe deer, blue sheep, and wild yak samples was seropositive for Brucella spp. The 16 samples that exhibited seropositivity to $C$. abortus were all from goitered gazelles, with a detection rate of $18.6 \%$ (95\% CI, 9.4-24.6\%), indicating that goitered gazelles may be a dominant animal species for $C$. abortus infection. Four wild ruminants had positive antibody signal for $C$. burnetii, an argali sheep, a blue sheep, a goitered gazelle, and a Siberian ibex, with detection rates of $18.8 \%(95 \%$ CI, 7-36\%), $10.0 \%$ (95\% CI, 1-32\%), $8.5 \%$ (95\% CI, $2.9-$ $14.2 \%$ ), and $13.3 \%$ (95\% CI, 4-31\%), respectively, for these animals. No antibodies to $C$. burnetii were detected in Tibetan antelope, roe deer, red deer, and wild yaks, indicating lower infection rates in these four wild ruminants. For toxoplasmosis, all six seropositive samples were from red deer, with a detection rate of $10.7 \%$ (95\% CI, 2.6-18.8\%).

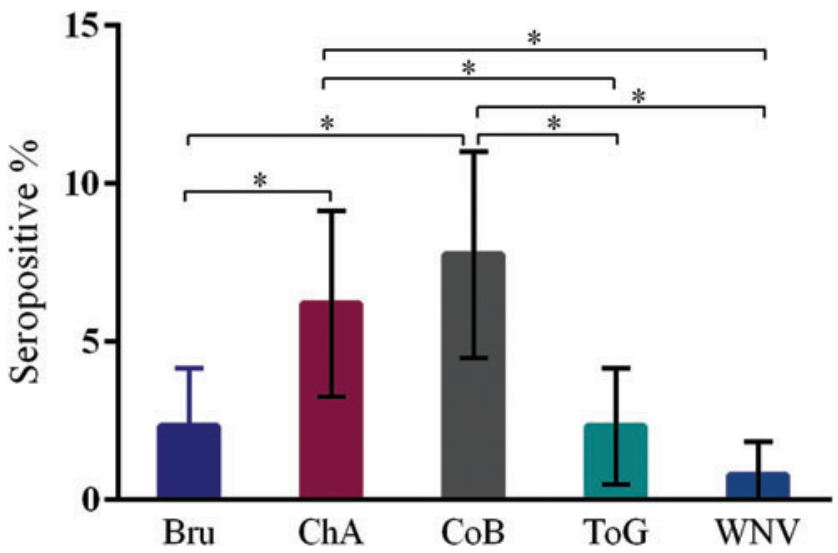

FIG. 2. Seroprevalence of the five zoonotic diseases in wild ruminants. $* p<0.05$. Bru, Brucella spp.; ChA, Chlamydophila abortus; CoB, Coxiella burnetii; ToG, Toxoplasma gondii; WNV, West Nile virus. Color images are available online.
Thus, at least in this region, red deer may be the dominant animal species for $T$. gondii infection. Two goitered gazelles exhibited anti-West Nile virus seropositivity, with a detection rate of $2.6 \%$ (95\% CI, $0-5.0 \%)$, while other wild ruminants were all seronegative (Table 2 ).

We next determined the geographic distribution of the five zoonotic diseases. Animals seropositive for one of the five zoonotic diseases were found in 8 out of 10 regions (all except Changji and Urumqi). Antibodies to Brucella spp. were found in Ili $(33.3 \%, 95 \%$ CI 4-78\%), Khotan $(9.1 \%, 95 \%$ CI 1-29\%), and Tacheng $(4.5 \%, 95 \%$ CI 1-15\%). Seropositivity to C. abortus was presented in Altay $(23.1 \%, 95 \%$ CI $11.6-34.5 \%)$ and Hami $(4.3 \%, 95 \%$ CI $0.2-8.5 \%)$. Wild ruminants infected with $C$. burnetii were identified in Aksu (50.0\%, 95\% CI 7-93\%), Hami (8.7\%, 95\% CI 2.9-14.5\%), Khotan (9.1\%, 95\% CI 0-21.1\%), Kashgar (16.7\%, 95\% CI $0-37.8 \%)$, and Tacheng $(13.6 \%, 95 \%$ CI $3.5-23.8 \%)$. Antibodies to T. gondii were detected in Altay $(3.8 \%, 95 \%$ CI 0-9.1\%), Bayingolin (28.6\% 95\% CI 8-58\%), and Tacheng $(13.6 \%, 95 \%$ CI 5-27\%). However, antibodies to West Nile virus were only found in Bayingolin (14.3\%, 95\% CI 2-43\%). In Tacheng, three out of the five zoonotic diseases were detected. In Altay, Bayingolin, Hami, and Khotan, two pathogens were identified, and in Aksu, Ili, and Kashgar, only one of the five zoonotic pathogens was detected (Table 2).

\section{Discussion}

Previous seroprevalence studies of animals identified some of these pathogens in domestic animals or focused on single pathogens (Lan et al. 2011, Li et al. 2018, Nie et al. 2018, Xing et al. 2018, Muñoz et al. 2019). This study examined eight species of wild ruminants and detected the seroprevalence of five zoonotic pathogens to understand the extent to which wild ruminants carry zoonotic pathogens. More than one-fifths of the animals tested were seropositive to at least one pathogen, indicating that wild ruminants are an important reservoir for zoonotic pathogens in Xinjiang, northwest China.

C. burnetii is a pathogen that causes $\mathrm{Q}$ fever, can infect a wide range of hosts, and is transmitted mainly by vectors (Eldin et al. 2017, Esmaeili et al. 2019). In this study, 7.8\% of the tested wild ruminants had antibodies to C. burnetii, which is similar to the rate previously reported in wildlife in Spain and free-range deer in the United States (Astobiza et al. 2011, de Souza Zanatto et al. 2019). Previous studies about $C$. burnetii infection in China focused on human and domestic animals, with significant variation in the detection of C. burnetii. For example, C. burnetii was found in $20.5 \%$ of cattle sampled in Xinjiang (Li et al. 2020), 24.9\% in sheep and $12.3 \%$ of Sika deer (Cong et al. 2015), and $4.7 \%$ of goats in Hubei province, China ( $\mathrm{Li}$ et al. 2018), indicating that C. burnetii infection varies for different animals and regions. Importantly, our results indicated that wild ruminants have relatively high infection with $C$. burnetii, suggesting that these animals are important carriers for $C$. burnetii. the diseased animal was infected by contact of $C$. burnetii spores or $C$. burnetii contaminated aborted materials (Gürtler et al. 2014), current efforts to monitor $C$. burnetii have mainly focused on vectors such as ticks and mites (Raele et al. 2018, Bártová et al. 2019). Diseased animals can be infected by 


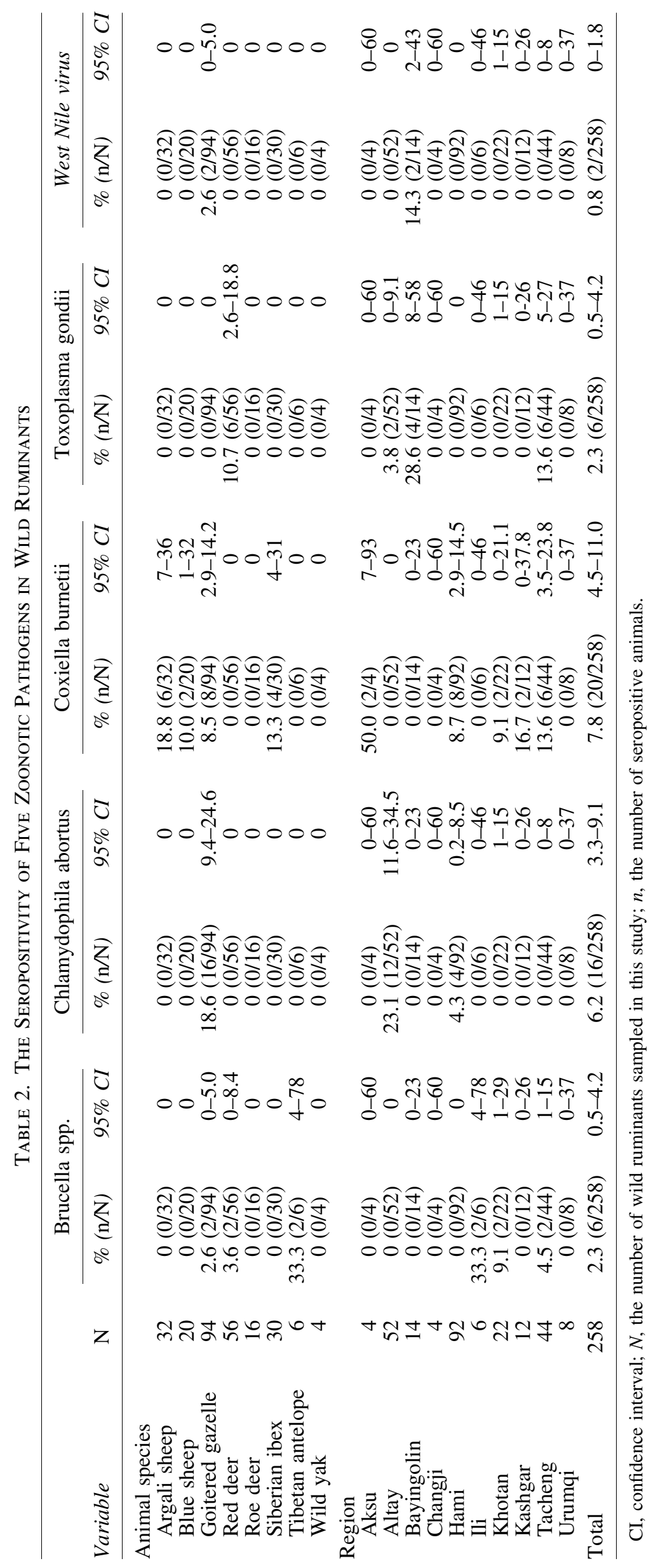


aerosolization of $C$. burnetti spores or by contact with materials contaminated with $C$. burnetti (Gürtler et al. 2014). The results from this work suggest that we should identify potential sources of infection (wildlife, domestic animals, or vectors) allowing transmission of $C$. burnetii to wild ruminants.

Brucellosis is a significant zoonotic disease that has been epidemic in parts of northern China. In this study, $2.3 \%$ seropositivity to Brucella spp. was detected in wild ruminants. During the same period, $2.4 \%$ of ovine and caprine and about $3.3 \%$ of dairy cattle tested positive for brucellosis in Xinjiang (Ran et al. 2018, 2019), indicating that wild ruminants may be an important reservoir for Brucella spp. The test kit antibody to Brucella spp. used here does not differentiate different species of Brucella. Some Brucella spp., such as Brucella melitensis, Brucella abortus, and Brucella canis, are very infectious to human, while others are less of a threat (Galińska and Zagórski, 2013). Therefore, characterizing and tracing Brucella spp. isolates and blocking transmission between wildlife and domestic livestock should be a priority for veterinary public health.

West Nile virus was previously isolated in human and mosquitoes in Xinjiang (Lu et al. 2011, Li et al. 2013), and serologic evidence was reported for dogs and cats in Shanghai (Lan et al. 2011, 2012), but not detected in other animals or vectors in China. West Nile virus outbreaks have occurred in birds, horses, and humans, and identification of West Nile virus in goitered gazelles in Xinjiang suggests that there might be other transmission routes for the spreading of West Nile virus.

T. gondii is a ubiquitous apicomplexan parasite in warmblooded animals and humans (Blader et al. 2015). A previous study reported $23.7 \%$ seroprevalence of $T$. gondii in food animals (sheep, goats, swine, chickens, and cattle) (Dong et al. 2018), and $20.5 \%$ seroprevalence was determined in horse in Xinjiang (Wang et al. 2015, Xing et al. 2018). The seroprevalence in red deer measured in this study was relatively lower than reported for horse, indicating both species can be hosts of $T$. gondii and should be monitored.

C. abortus is an obligate intracellular gram-negative bacteria, that can infect animals and cause abortion worldwide (Longbottom et al. 2018). C. abortus infection has mainly been reported in farmed animals in China, such as goats, white yaks, pig, wild boars, fur animals, and rabbits (Ni et al. 2015, Qin et al. 2015, Li et al. 2018, Nie et al. 2018, Sun et al. 2019). In this study, C. abortus was detected at $18.6 \%$, a rate that is similar to that reported for white yaks and sika deer, but lower than that in farmed wild boars (Qin et al. 2015, Liu et al. 2018, Nie et al. 2018). C. abortus was only detected in goitered gazelles, suggesting that these animals may be important wild carriers in this region.

These five zoonotic pathogens are commonly found in domestic livestock, and efforts to block the transmission chain from domestic animals and wildlife should be a priority of veterinary departments to meet the overall health requirements at the human-animal-environment interface advocated by the One Health concept (Mwangi et al. 2016), designed to limit transmission from wildlife to domestic animals and even humans. In efforts to stop a Mycobacterium bovis epidemic, scientists tried to identify the key species that were infected, since the effective control of pathogens in wildlife is key for comprehensive control of zoonotic pathogens (Gowtage et al. 2017). Overall, we suggest that vet- erinary and human public health departments should strongly consider the risks of a zoonotic pathogen epidemic in Xinjiang, control and monitor of zoonotic diseases in wild animal populations, and develop oral vaccines against zoonotic pathogens. In addition, efforts should be made to prohibit grazing in nature reserves to reduce opportunities for livestock to contact wild animals, which can limit the spilling of zoonotic pathogens from wild ruminants into domestic animals and even humans.

In conclusion, seropositivity to zoonotic pathogens is common among wild ruminants in Xinjiang, Northwest China, with the highest reactivity observed for $C$. burnetii and $C$. abortus. This study provides the baseline to assess the potential for spillover events of zoonotic diseases.

\section{Acknowledgment}

We are indebted to Prof. Zhi-Cai Wang, who retired from Institute of Veterinary Medicine, Xinjiang Academy of Animal Sciences, for his kind guidance for sample collection.

\section{Author Disclosure Statement}

No conflicting financial interests exist.

\section{Funding Information}

This work was supported by the National Key Research and Development Program of China (grant no. 2017YFD0501802).

\section{Supplementary Material}

Supplementary Appendix Table S1

\section{References}

Astobiza I, Barral M, Ruiz-Fons F, Barandika JF, et al. Molecular investigation of the occurrence of Coxiella burnetii in wildlife and ticks in an endemic area. Vet Microbiol 2011; 147:190-194.

Bártová E, Kučerov HL, Žákovská A, Budíková M, et al. Coxiella burnetii and Francisella tularensis in wild small mammals from the Czech Republic. Ticks Tick-Borne Dis 2019; 11:101350.

Belay ED, Kile JC, Hall AJ, Barton-Behravesh C, et al. Zoonotic disease programs for enhancing global health security. Emerg Infect Dis 2017; 23:S65-S70.

Blader IJ, Coleman BI, Chen C-T, Gubbels M-J. Lytic cycle of Toxoplasma gondii: 15 years later. Annu Rev Microbiol 2015; 69:463-485.

Cong W, Meng Q-F, Shan X-F, Sun W-W, et al. Coxiella burnetii ( $\mathrm{Q}$ fever) infection in farmed ruminants in three northeastern provinces and inner Mongolia autonomous region, China. Vector Borne Zoonotic Dis 2015; 15:512-514.

de Souza Zanatto DC, Duarte JMB, Labruna MB, Tasso JB, et al. Evidence of exposure to Coxiella burnetii in neotropical free-living cervids in South America. Acta Trop 2019; 197: 105037.

Dong H, Su R, Lu Y, Wang M, et al. Prevalence, risk factors, and genotypes of Toxoplasma gondii in food animals and humans (2000-2017) from China. Front Microbiol 2018; 9 : 2108 . 
Eldin C, Mélenotte C, Mediannikov O, Ghigo E, et al. From Q fever to Coxiella burnetii infection: A paradigm change. Clin Microbiol Rev 2017; 30:115.

Escribano-Romero E, Lupulovic D, Merino-Ramos T, Blazquez $\mathrm{AB}$, et al. West Nile virus serosurveillance in pigs, wild boars, and roe deer in Serbia. Vet Microbiol 2015; 176:365-369.

Esmaeili S, Mobarez AM, Khalili M, Mostafavi E, et al. Molecular prevalence of Coxiella burnetii in milk in Iran: A systematic review and meta-analysis. Trop Anim Health Prod 2019; 51:1-11.

Galińska E, Zagórski J. Brucellosis in humans-Etiology, diagnostics, clinical forms. Ann Agr Env Med 2013; 20:233238.

Gowtage S, Williams GA, Henderson R, Aylett P, et al. Testing of a palatable bait and compatible vaccine carrier for the oral vaccination of European badgers (Meles meles) against tuberculosis. Vaccine 2017; 35:987-992.

Gürtler L, Bauerfeind U, Blümel J, Burger R, et al. Coxiella burnetii-Pathogenic agent of Q (Query) Fever. Transfus Med Hemother 2014; 41:60-72.

Jennelle CS, Carstensen M, Hildebrand EC, Cornicelli L, et al. Surveillance for highly pathogenic avian influenza virus in wild birds during outbreaks in domestic poultry, Minnesota, 2015. Emerg Infect Dis 2016; 22:1278.

Lan D, Ji W, Yu D, Chu J, et al. Serological evidence of West Nile virus in dogs and cats in China. Arch Virol 2011; 156: 893-895.

Lan D, Wang C, Deng B, Zhou J, et al. Serological investigations on West Nile virus in birds and horses in Shanghai, China. Epidemiol Infect 2012; 141:1-5.

Li K, Luo H, Shahzad M. Epidemiology of Q-fever in goats in Hubei province of China. Trop Anim Health Prod 2018; 50: 1395-1398.

Li M, Liu H, Bi Y, Sun J, et al. Highly pathogenic avian influenza $\mathrm{A}(\mathrm{H} 5 \mathrm{~N} 8)$ virus in wild migratory birds, Qinghai Lake, China. Emerg Infect Dis 2017; 23:637-641.

Li X-L, Fu S-H, Liu W-B, Wang H-Y, et al. West Nile virus infection in Xinjiang, China. Vector Borne Zoonotic Dis 2013; 13:131-133.

Li Y, Li J, Chahan B, Guo Q, et al. Molecular investigation of tick-borne infections in cattle from Xinjiang Uygur Autonomous Region, China. Parasitol Int 2020; 74:101925.

Liu F, Li J-M, Zeng F-L, Zong Y, et al. Prevalence and risk factors of brucellosis, Chlamydiosis, and bluetongue among sika deer in Jilin Province in China. Vector Borne Zoonotic Dis 2018; 18:226-230.

Longbottom D, Sait M, Livingstone M, Laroucau K, et al. Genomic evidence that the live Chlamydia abortus vaccine strain $1 \mathrm{~B}$ is not attenuated and has the potential to cause disease. Vaccine 2018; 36:3593-3598.

Lu Z, Fu S-H, Cao L, Tang C-J, et al. Human infection with West Nile virus, Xinjiang, China, 2011. Emerg Infect Dis 2011; 20:1421-1423.

Menachery VD, Yount BL, Jr., Sims AC, Debbink K, et al. SARS-like WIV1-CoV poised for human emergence. Proc Natl Acad Sci U S A 2016; 113:3048-3053.

Miernyk KM, Bruden D, Parkinson AJ, Hurlburt D, et al. Human Seroprevalence to 11 zoonotic pathogens in the U.S. Arctic, Alaska. Vector Borne Zoonotic Dis 2019; 19:563-575.

Muñoz PM, Mick V, Sacchini L, Janowicz A, et al. Phylogeography and epidemiology of Brucella suis biovar 2 in wildlife and domestic swine. Vet Microbiol 2019; 233:68-77.
Mwangi W, de Figueiredo P, Criscitiello MF. One health: Addressing global challenges at the nexus of human, animal, and environmental health. PLoS Pathog 2016; 12:e1005731.

Ni X, Qin S, Lou Z, Ning H, et al. Seroprevalence and risk factors of Chlamydia infection in domestic rabbits (Oryctolagus cuniculus) in China. Biomed Res Int 2015; 2015: 460473.

Nie L-B, Liang Q-L, Zou Y, Gao Y-H, et al. First report of Chlamydia seroprevalence in farmed wild boars in China. Vector Borne Zoonotic Dis 2018; 18:212-214.

Petrosillo N. Emerging infections and future threats. Erciyes Med J 2019; 41:130-135.

Qin S-Y, Huang S-Y, Yin M-Y, Tan Q-D, et al. Seroprevalence and risk factors of Chlamydia abortus infection in freeranging white yaks in China. BMC Vet Res 2015; 11:8.

Raele D, Galante D, Pugliese N, La Salandra G, et al. First report of Coxiella burnetii and Borrelia burgdorferi sensu lato in poultry red mites, Dermanyssus gallinae (Mesostigmata, Acari), related to urban outbreaks of dermatitis in Italy. New Microbes New Infect 2018; 23:103-109.

Ran X, Chen X, Wang M, Cheng J, et al. Brucellosis seroprevalence in ovine and caprine flocks in China during 2000-2018: A systematic review and meta-analysis. BMC Vet Res 2018; 14:393.

Ran X, Cheng J, Wang M, Chen X, et al. Brucellosis seroprevalence in dairy cattle in China during 2008-2018: A systematic review and meta-analysis. Acta Trop 2019; 189: 117-123.

Steyn J, Botha E, Stivaktas VI, Buss P, et al. West Nile virus in wildlife and nonequine domestic animals, South Africa, 2010-2018. Emerg Infect Dis 2019; 25:2290-2294.

Sun W-W, Zhang N-Z, Kang Y-H, Zhang L, et al. First Report of Chlamydia seroprevalence in slaughter pigs in Shandong Province, Eastern China. Vector Borne Zoonotic Dis 2019; 20:51-53.

Tomassone L, Berriatua E, De Sousa R, Duscher GG, et al. Neglected vector-borne zoonoses in Europe: Into the wild. Vet Parasitol 2018; 251:17-26.

Wang J-L, Zhou D-H, Chen J, Liu G-X, et al. The prevalence of antibodies to Toxoplasma gondii in horses in Changji Hui Autonomous Prefecture, Xinjiang, northwestern China. Rev Bras Parasitol Vet 2015; 24:298-302.

Wei Y-R, Yang X-Y, Li Y-G, Wei J, et al. Serological survey of avian influenza virus infection in non-avian wildlife in Xinjiang, China. Arch Virol 2016; 161:867-872.

Xia J, Zheng X, Adili G, Wei Y, et al. Sequence analysis of peste des petits ruminants virus from ibexes in Xinjiang, China. Genet Mol Res 2016; 15:gmr.15027783.

Xing H, Xu L, Song X, Li X, et al. Seroprevalence of Toxoplasma gondii and Trichinella spiralis in Horses in Xinjiang, Northwestern China. J Equine Vet Sci 2018; 60:11-15.

Address correspondence to: Xue-Yun Yang

Institute of Veterinary Medicine Xinjiang Academy of Animal Sciences No.726 Dongrong Street Urumqi 830013

China

E-mail: yangxyxj@foxmail.com 